Revista Médica Sinergia Vol.3 Num:9

Setiembre 2018 pp: 14 - 19 ISSN:2215-4523 e-ISSN:2215-5279

http://revistamedicasinergia.com

\section{SÍNDROME DE VENA CAVA SUPERIOR: URGENCIA ONCOLÓGICA}

(Superior vena cava syndrome: oncological emergency)

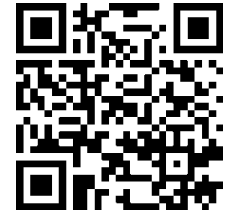

${ }^{1}$ Medicina general, graduada de la Universidad de Ciencias Médicas (UCIMED), médico del Hospital San Juan de Dios en el servicio de Hematooncología. San José, Costa Rica. Código médico: 13642 naniddelgado@gmal.com

\author{
${ }^{1}$ Dra. Dahiana Delgado Delgado \\ Hospital San Juan de Dios, San José, Costa Rica \\ naniddelgado@gmal.com \\ https://orcid.org/0000-0002-5004-383X
}

DOI: https://doi.org/10.31434/rms.v3i9.139

\section{RESUMEN}

La primera descripción del síndrome de vena cava superior se realizó en 1757, por un médico escoces, William Hunter, en un paciente con aneurisma aórtico por sífilis. Se le da ese nombre a cualquier condición que produzca obstrucción del flujo sanguíneo en la vena cava superior. Con el paso del tiempo gracias a la instauración de antibióticos, las causas infecciosas fueron desplazadas por las neoplásicas (90\%) y en menor medida por artefactos intravenosos, en donde se produce una trombosis. El tratamiento depende de la etiología y de la gravedad, principalmente se utiliza la radioterapia y quimioterapia en casos de etiología maligna. Este síndrome se convierte en una verdadera emergencia cuando produce compresión de la vía aérea.

PALABRAS CLAVES: urgencia, oncología, síndrome, vena cava superior.

\section{ABSTRACT}

The first description of superior vena cava syndrome was performed in 1757 , by a Scottish physician, William Hunter, in a patient with syphilis aortic aneurysm. Any condition that causes obstruction of blood flow in the superior vena cava is given this name. Over time antibiotics were introduced, the infectious causes was displaced by neoplastic ones (90\%) and to a lesser extent by intravenous devices, where thrombosis occurs. Treatment depends on etiology and severity, mainly radiotherapy and chemotherapy in cases of malignan etiology. This syndrome becomes a real emergency when it produces compression of the airway.

KEY WORDS: urgency, oncology, syndrome, superior vena cava. 


\section{INTRODUCCIÓN}

Las urgencias oncológicas se dividen en tres grandes grupos:

1. Obstrucciones producidas por una lesión espacio-ocupante

2. Trastornos metabólicos

3. Complicaciones generales por causa del tratamiento

La vena cava superior se origina de la unión de las dos venas braquicefálicas a nivel del mediastino superior, justo detrás del primer cartílago costal derecho, con una longitud aproximada de $6 \mathrm{~cm} \mathrm{y}$ desemboca en la aurícula derecha; es el principal vaso para el drenaje venoso de la cabeza, cuello, miembros superiores y parte superior del tórax, llevando cerca de un tercio del total de sangre venosa al corazón.

\section{FISIOPATOLOGÍA}

Las paredes de la vena cava superior son delgadas y fáciles de comprimir, por lo tanto vulnerable a que cualquier crecimiento de las estructuras adyacentes la obstruya, como es el caso de los ganglios linfáticos que la rodean. Cuando se da una compresión $u$ obstrucción de la vena cava superior, la sangre retorna por venas colaterales como la vena ácigos, que desemboca en la parte posterior de la vena cava superior, en el caso en que la compresión se da por debajo de la desembocadura de la vena ácigos la sangre intentara retornar por la vena cava inferior y esto supone una mayor presión venosa; otros sistemas de drenaje colaterales son la vena mamaria interna, vena torácica lateral, vena esofágica; a estas venas les toma algún tiempo poder dilatarse lo suficiente para manejar el aumento de volumen lo que explica que las presiones venosas sean mayores al inicio del cuadro; la presión venosa cervical generalmente es de 2 a $8 \mathrm{mmHg}$, pero al producirse una obstrucción esta presión alcanza niveles de 20 a $40 \mathrm{mmHg}$.

\section{ETIOLOGÍA}

Antes de los avances en los antibióticos, las principales causas eran infecciosas, como el aneurisma de aorta torácica sifilítico, la mediastinitis fibrosante y tuberculosis; posteriormente las causas malignas tomaron el primer lugar, actualmente corresponden un $90 \%$ de las causas del síndrome de vena cava superior, y de estas la primera causa es el cáncer de pulmón.

\section{CÁNCER DE PULMÓN}

Entre un $2-4 \%$ de los pacientes con cáncer pulmonar van a desarrollar síndrome de vena cava superior en algún momento de la evolución de la enfermedad. El tipo histológico más frecuente es el carcinoma no microcítico de pulmón responsable de un $50 \%$ de las obstrucciones y tiende a ubicarse en la periferia del pulmón, en segundo lugar está el carcinoma microcítico, con un $25 \%$ de los casos, su menor incidencia se debe a su localización y rápido crecimiento en la parte central de las vías respiratorios, no es común verlo en la periferia del pulmón. En estos pacientes la obstrucción es en su mayoría extrínseca por compresión del tumor o de los ganglios linfáticos con enfermedad metastásica, y en la minoría de los casos por una invasión a la vena cava superior. 


\section{LINFOMA NO HODGKIN}

Se le atribuye alrededor del $10-15 \%$ de los casos, los subtipos más frecuentes son: el linfoma primario mediastínico de células $B$ grandes, que es el subtipo más común, se presenta en un $60 \%$ de los casos por linfoma; el linfoma linfoblástico y el linfoma folicular de células grandes.

\section{METÁSTASIS}

Representan un pequeño porcentaje, corresponden principalmente de tumores germinales y cáncer de mama.

\section{ARTEFACTOS INTRAVENOSOS}

En muchas ocasiones los pacientes oncológicos requieren de la colocación de catéteres venosos centrales, para la administración frecuente de quimioterapia, antibióticos o tratamientos sintomáticos, esto propicia la formación de trombos por la injuria en el endotelio; además los procesos oncológicos crean un ambiente pro coagulante.

\section{PRESENTACIÓN CLÍNICA}

Los signos y síntomas dependen del grado y de la rapidez en el inicio de la obstrucción, siendo más floridos en las primeras semanas cuando los vasos colaterales aún no se han dilatado completamente; en un tercio de los pacientes los síntomas tienden a aparecer dentro de las primeras 2 semanas, en otros casos toma más tiempo poder ver las manifestaciones clínicas. Ver TABLA 2.1.

La severidad de los síntomas determinará el grado de urgencia con que se debe tratar al paciente.

\begin{tabular}{|l|c|}
\hline \multicolumn{1}{|c|}{ SIÍNTOMAS } & $\begin{array}{c}\% \\
\text { PACIENTES }\end{array}$ \\
\hline Disnea & $60 \%$ \\
\hline $\begin{array}{l}\text { Sensación de aumento de } \\
\text { volúmen facial y el cuello }\end{array}$ & $50 \%$ \\
\hline Tos & $24 \%$ \\
\hline Inflamación en brazos & $18 \%$ \\
\hline Dolor en el pecho & $15 \%$ \\
\hline Disfagiagia & $9 \%$ \\
\hline & $\%$ \\
\hline Distención venas del cuello & $60 \%$ \\
\hline Venas colaterales en tórax & $54 \%$ \\
\hline Edema facial & $46 \%$ \\
\hline Cianosis & $20 \%$ \\
\hline Edema en brazos & $14 \%$ \\
\hline $\begin{array}{l}\text { Adaptado del Devita V, Lawrence T, Rosenberg S. } \\
\text { "Devita, Hellman and Rosenberg's Cancer Principles \& } \\
\text { Practice of Oncology". 10th edition. }\end{array}$ \\
\hline
\end{tabular}

Las manifestaciones no se producen solo por el aumento de la presión hidrostática en la vena cava superior, sino también, por la compresión o invasión de las vías respiratorias 0 los nervios. Frecuentemente los pacientes perciben una sensación de aumento de volumen en el cuello y la cara que se exacerba cuanto se inclinan hacia adelante, además de disnea y tos.

Los signos característicos son:

- Dilatación de las venas cervicales

- Presencia de venas colaterales en la parte superior del tórax

- Edema en cuello y cara (principalmente en la región periorbitaria)

En los casos graves se presentan con edema laríngeo, lingual, síntomas de edema cerebral, en estos casos se debe 
realizar una valoración urgente; en los casos donde se presente convulsiones se relacionaría más con metástasis cerebrales que con el síndrome de vena cava superior.

En raras ocasiones se presenta varices esofágicas con un sentido cefálicocaudal eso sería un diagnóstico diferencial de las varices por hipertensión portal, que presentan en sentido caudalcefálico. Los síntomas son más solapados cuando la obstrucción ocurre antes de la desembocadura de la vena ácigos.

\section{DIAGNÓSTICO}

El diagnóstico es sobre todo clínico, pero se debería realizar una seria de estudios de imagen y correlación histológica, para garantizar un correcto tratamiento.

\section{- IMÁGENES}

Son el pilar de la confirmación del diagnóstico, en la mayoría de los casos se realiza una tomografía de tórax (TAC) con medio de contraste que presenta una alta sensibilidad, alrededor de $92 \%$, además proporción información sobre estructuras de gran importancia, como la tráquea. La venografía por resonancia magnética es la mejor opción en pacientes alérgicos al contraste.

\section{- HISTOLOGÍA}

Antes de iniciar un tratamiento con radioterapia o con quimioterapia, hay que realizar una confirmación histológica, ya sea a través de un aspirado con aguja fina endobronquial guiado por TAC, o en casos donde no se pueda realizar el aspirado se recomienda realizar una mediastinoscopia diagnostica o en última instancia una toracotomía exploratoria con toma de biopsia. En casos que se presenten con derrame pleural se puede realizar una toracocentesis diagnostica.

\section{TRATAMIENTO}

El objetivo del tratamiento en el síndrome de vena cava superior asociado a etiología maligna es aliviar los síntomas, y tratar la enfermedad subyacente, por ende, dependerá de la extensión del tumor, la histología y la administración previa de tratamientos. En su mayoría se utiliza la quimioterapia, radioterapia 0 una combinación de ambos. En los casos que cursen con estridor, debido a una obstrucción importe de las vías aéreas, o se presente con edema cerebral, se requiere tratamiento inmediato con radioterapia o con la colocación de un stent, ya que pone en riesgo inminente de muerte al paciente.

\section{- RADIOTERAPIA}

Se recomienda en paciente con tumores radiosensibles, principalmente en el cáncer de pulmón; y en casos que no hayan recibido radioterapia previamente. Cuando se utiliza proporciona un alivio importante al reducir la carga tumoral. Antes de iniciar este tratamiento se recomienda contar con una biopsia del tejido, ya que después de la radioterapia no se podrá obtener un diagnostico histológico.

\section{- QUIMIOTERAPIA}

Es el tratamiento inicial de elección para los linfomas No-Hodgkin y tumores de células germinales. El alivio completo de los síntomas se logra en un $80 \%$ en los pacientes con Linfoma No-Hodgkin. En los pacientes con Carcinoma de células pequeñas de pulmón el tratamiento 
preferido es la quimioterapia, aunque se han presentados estudios utilizando quimioterapia combinado con radioterapia con una respuesta exitosa.

\section{CONCLUSIÓN}

El síndrome de vena vaca superior se considera una emergencia médica cuando se presenta con una obstrucción importe de las vías aéreas o curse con edema cerebral, al presentar síntomas cerebrales se debe realizar un diagnóstico diferencial con metástasis cerebrales. La intensidad de los síntomas dependerá del nivel y grado de obstrucción de la vena cava superior. Las causas más frecuentes son neoplásicas y en menor medida por trombos. El tratamiento de basa en la histología del tumor, la mayoría de los tumores son radiosensibles, principalmente los de Pulmón; en los linfomas No-Hodgkin y cáncer de células germinales la primera elección es la quimioterapia. La sobrevida estimada en pacientes oncológicos con este síndrome es de seis meses, pero es muy relativa según las características histológicas.

\section{BIBLIOGRAFÍA}

1. Casciato D, Territo Mary. "Manual de Oncología Clínica". 7ma Edición. Lippincott Williams \& Wilkins. España. 2014. Pp 696-700.

2 Devita V, Lawrence T, Rosenberg S. "Devita, Hellman and Rosenberg's Cancer Principles \& Practice of Oncology". 10th edition. Wolters Kluwer Health. USA .2015. Pp 1804-1809.

3. Drews RE, Rabkin DJ. (2016). Malignancy-related superior vena cava syndrome. UpToDate, Waltham, MA.

4. Khan U, Shanholtz C, y McCurdy M. (2014) “Oncologic Mechanical Emergencies". Emergency Medicine Clinics of North America, 32(3): 495-508.

5. Latarjet M, Liard R. "Anatomía Humana". 4ta Edición. Editorial medica panamericana. Buenos Aires, Argentina. 2005. Pp 1037-1057.

6. Lepper P, Ott S, Hoppe H, et al. (2011). "Superior Vena Cava Syndrome in Thoracic Malignancies". Respiratory Care, 58(5): 653-666.

7. Longo D, Kasper D, Jameson J, Fauci A, Hauser S, Loscalzo J. "Harrison Principios de Medicina Interna”. 18ª edición. McGraw-Hill Interamericana. México, D.F. 2012. Pp 2266-2267.

8. McCurdy M, y Schanholtz C. (2012) "Oncologic Emergencies". Critical Care Medicine, 40(7): 2212-2222.

9. Rachapalli V, y Boucher LM. (2014) "Superior Vena Cava Syndrome: Role of the Interventionalist". Canadian Association of Radiologist Journal, 65(2): 168-176. 
10. Rodríguez JL, et al. (2013). Green Book Diagnóstico y Tratamiento Médico. Madrid, España: MARBÁN. Pp: 136-137.

11. Talapatra K, Panda S, Goyle S, et al. (2016) "Superio Vena Cava Syndrome:A radiation oncologist's perspective”. Journal of Cancer Research and Therapeutics, 12(2):515-519.

12 Wilson L, Detterbeck F, Yahalom J. (2007) "Superior Vena Cava Syndrome with Malignant Cause". New England journal of medicine, 356(18): 1862-1869.

Recepción: 13 Junio de 2018

Aprobación: 10 Agosto de 2018 